\title{
Diabtesrisiko Schlafentzug
}

\section{Kurze Schlafdauer verschlechtert die Glukosetoleranz}

Schon kurzfristiger Schlafentzug führt messbar zu einer Verschlechterung der Glukosetoleranz durch verminderte Insulinsensitivität. Das ergab eine Studie mit 15 gesunden, normalgewichtigen Männern an der Universität Lübeck. Nach kurzer Bettruhe (2.45 bis $7.00 \mathrm{Uhr}$ ) waren die Spitzenwerte für Insulin und Glukose deutlich höher als nach normaler ab 22.45 Uhr. Bei allen Teilnehmern, die auch Kontrollen waren, wurde jeweils nach zwei Nächten eingeschränkter bzw. normaler Bettruhe Folgendes 15 Stunden lang eng überwacht: Glukose, Insulin, C-Peptid, Glukagon, ACTH, Cortisol, IL-6.

Ergebnisse: Nach Schlafentzug waren vor dem Frühstück die Konzentrationen der ausgewählten Blutpara- meter unverändert $(\mathrm{p}>0,30)$. Nach dem Frühstück um 8 Uhr waren jedoch die Spitzenwerte für Insulin und Glukose nach dem Schlafentzug versus nach normalem Schlaf deutlich erhöht (Insulin: 398,5 \pm 57,4 im Vergleich zu 284,3 $\pm 51,5 \mathrm{pmol} / \mathrm{l}$ bzw. Glukose: 6,8 \pm 0,3 im Vergleich zu 6,1 $\pm 0,3 \mathrm{mmol} / \mathrm{l}$, jeweils $\mathrm{p}<0,02$ ), der Glukagon-Wert wurde durch den Schlafentzug reduziert $(\mathrm{p}=$ 0,03). Bei zirkulierendem ACTH, Cortisol und IL-6 gab es keine Unterschiede. Die Glukosetoleranz ist offenbar v.a. durch reduzierte Insulinsensitivität verschlechtert. Die unveränderte Hypothalamus-Hypophysen-Nebennierenrinden-Achse sowie IL-6-Konzentration sprechen dagegen, dass diese Effekte auf stress- oder entzündungsbedingte Mechanismen zurückzuführen sind.

\section{Übergewicht durch Schlafmangel Schlaf reguliert den Energieverbrauch}

Es ist bekannt, dass chronischer Schlafmangel das Risiko für Übergewicht erhöht, aber nicht warum. Eine Studie legt nahe, dass Schlaf sich regulierend auf den Energieverbrauch auswirkt. Denn Teilnehmer mit völligem Schlafentzug hatten einen signifikant reduzierten Energieverbrauch im Ruhezustand und nach dem Essen. In der Querschnittsstudie wurden 14 normalgewichtige Männer zweimal 24 Stunden lang beobachtet: einmal bei normalem Schlaf-Wach-Zyklus mit acht Stunden nächtlichen Schlafs und einmal während einer 24-stündigen Wachperiode. Jeweils am Morgen danach wurde mithilfe indirekter Kalorimetrie der Energieverbrauch im Ruhezustand sowie nach dem Essen ermittelt. Zudem wurde danach dokumentiert, welche Speisen die Probanden von einem reichhaltigen Buffet wählten.

Ergebnisse: Im Vergleich zu der Nacht mit normalem Schlaf waren nach der Nacht mit Schlafentzug der Energieverbrauch in Ruhe um ca. 5\% und nach dem Essen um ca. $20 \%$ signifikant reduziert. Ghrelin, Thyreotropin, Cortisol und Norepinephrin am Morgen und der Glukosespiegel nach dem Essen waren ebenfalls erhöht.

\section{LDL-Cholesterinsenkung...}

\section{...klappt auch ohne Arznei}

Sowohl blutfettsenkende Medikamente als auch positive Veränderungen des Lebensstils (mehr Bewegung, Ernährungsumstellung) führten unabhängig voneinander zu einer signifikanten Verringerung des LDL-Cholesterins. Dies bestätigt Präventionsstrategien, die auf breitgefächerten Interventionsmaßnahmen basieren. Das zeigen Auswertungen der Whitehall-II-Studie (Universität London), an der 4469 britische Beamte (Männer und Frauen) im Alter von zu Beginn 39 bis 62 Jahren durchschnittlich 11,3 Jahre lang teilgenommen haben.

Ergebnisse: Die mittlere LDL-Konzentration sank im Beobachtungszeitraum von 4,38 auf $3,52 \mathrm{mmol} / \mathrm{l}$. In einem wechselseitig adjustierten Modell nahm das LDL-

Cholesterin bei Personen, die schon zu Beginn der Studie Lipidsenker einnahmen $(-1,14 \mathrm{mmol} / \mathrm{l}, \mathrm{n}=34)$ bzw. im Verlauf mit deren Einnahme begannen $(-1,77$ $\mathrm{mmol} / \mathrm{l}, \mathrm{n}=481) \mathrm{im}$ Vergleich zu Personen ohne diese Medikamente $(\mathrm{n}=3954)$ signifikant mehr ab. Nach Ernährungsumstellung - v.a. das Verhältnis von weißem bzw. rotem Fleisch sowie von mehrfach un-/gesättigten Fettsäuren wurde geändert - sank das LDL (-0,07 $\mathrm{mmol} / \mathrm{l}, \mathrm{n}=717)$, - im Vergleich zu Personen ohne Umstellung $(n=3071)$ ebenfalls signifikant mehr $(p=0,03)$, und auch bei jenen, die sich verstärkt körperlich betätigten $(-0,10 \mathrm{mmol} / \mathrm{l}, \mathrm{n}=601)$, im Vergleich zu Personen, die ihr Sportverhalten nicht veränderten $(n=3312$; $\mathrm{p}=0,005)$. Quelle (3 Texte): www.lifestyle-telegramm.de
Schmid SM, Hallschmid M, JauchChara Ket al. Sleep 2011 1;34(3):371-7.

Benedict C, Hallschmid M, Lassen A et al. Am J Clin Nutr. 2011;93(6):122936.

Bouillon K, SinghManoux A, Jokela $M$ et al. Heart. 2011;97(11):923-30.

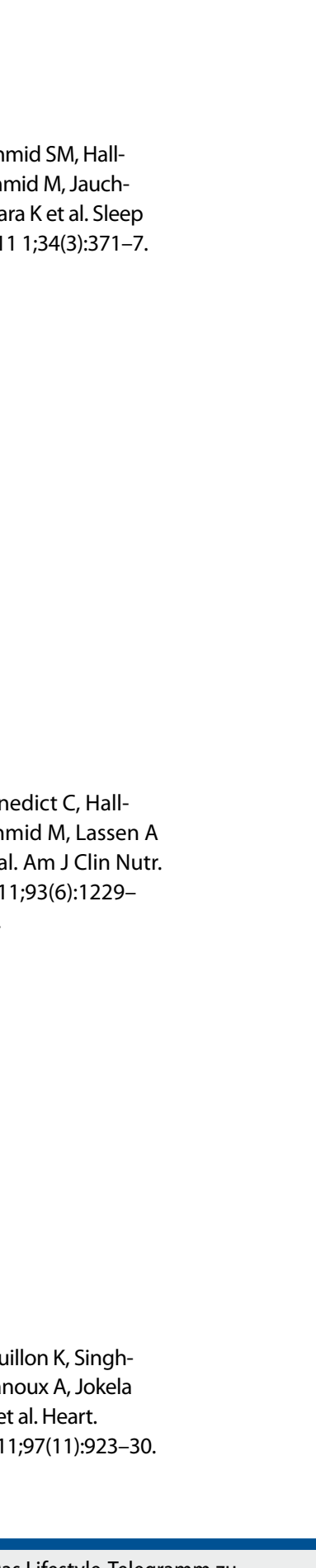

Das Lifestyle-Telegramm zu hochwertigen Publikationen mit dem Thema "Lebensstil" kann als Newsletter kostenlos abonniert werden und erscheint monatlich per Mail. Viele weitere entsprechende Kurzreferate gibt's unter: www.lifestyle-telegramm.de 